\title{
Homocysteine and methylmalonic acid concentrations in cerebrospinal fluid: relation with age and Alzheimer's disease
}

\author{
J-M Serot, F Barbé, E Arning, T Bottiglieri, P Franck, P Montagne, J-P Nicolas
}

J Neurol Neurosurg Psychiatry 2005;76:1585-1587. doi: 10.1136/jnnp.2004.056119

Background: Homocysteine may be involved in the pathogenesis of late onset Alzheimer's disease. It is implicated in the metabolism of several important pathways in the brain. Methylmalonic acid (MMA) is related to the metabolism of branched chained amino acids and fatty acids.

Objectives: To compare cerebrospinal fluid (CSF) total homocysteine and MMA in elderly subjects, patients with Alzheimer's disease, and younger healthy controls.

Subjects: CSF samples were obtained from 33 patients under 20 years of age; 28 patients aged 21 to 60 years; 22 normal elderly subjects aged over 60; and 38 Alzheimer patients aged over 60.

Results: CSF total homocysteine increased with age (mean (SD): 57 (35) nmol/l in the youngest group v 123 (89) nmol/l in the elderly group $(p<0.001))$ There was no difference between the elderly group and Alzheimer patients (1 15 (62) $\mathrm{nmol} / \mathrm{l})$. CSF MMA did not differ in the elderly group and the Alzheimer group (38 (13) v 35 (14) ng/ml). In the youngest group, it was significantly higher (60 (15) ng/ml).

Conclusions: CSF total homocysteine is not increased in Alzheimer's disease compared with age matched controls. CSF total homocysteine was correlated with age. The decrease in CSF MMA levels with age eliminates a lack of vitamin B-12 at neuronal level.

$\mathrm{T}$ he frequency of Alzheimer's disease increases with age. Despite numerous studies, the pathogenesis remains poorly understood. Anatomically, it is characterised by the coexistence of two different types of abnormality: senile plaques, which are extracellular formations with a central compound primarily composed of protein amyloid; and neurofibrillary tangles, which are formed of intracellular paired helical filaments. During Alzheimer's disease, the brain is the seat of considerable oxidative stress. Recent studies have suggested that cardiovascular risk factors such as hypertension, diabetes, and plasma homocysteine could be involved in the pathogenesis of late onset Alzheimer's disease. ${ }^{1}$

Homocysteine is formed by demethylation of the essential amino acid methionine. This free methyl group is implicated in the synthesis of numerous brain substrates. ${ }^{2}$ Homocysteine is catabolised by two possible pathways: in the first, homocysteine and serine are condensed to form cystathionine; in the second, the remethylation of homocysteine to methionine is dependent on methionine synthase. Vitamin B-12 and folate are the cofactors of this enzyme.

Methylmalonic acid (MMA) is a dicarboxylic acid related to the metabolism of several amino acids. ${ }^{3}$ Vitamin B-12 is required as a cofactor in the conversion of methylmalonyl coA to succinyl coA. In vitamin B-12 deficiency, MMA coA is converted to $\mathrm{MMA}^{3}$ and results in an intracellular increase in
MMA followed by increased concentrations in the serum. Homocysteine and MMA are known to be the most sensitive serum markers of early intracellular vitamin B-12 and folate deficiency. ${ }^{45}$ Folate deficiency results in increased total homocysteine levels. ${ }^{2}$ In cobalamin deficiency, cerebrospinal fluid (CSF) levels of homocysteine and MMA are raised but the increase of CSF MMA concentration is more important than that of homocysteine. ${ }^{6}$

Several studies have also suggest a direct correlation between Alzheimer's disease and serum total homocysteine levels. ${ }^{1}$ Decreased brain and CSF vitamin B-12 and decreased CSF folate have been described in Alzheimer's disease. ${ }^{5-9}$ These abnormalities could be responsible for modifications to brain metabolism. The diagnosis of mild vitamin B-12 deficiency is often difficult. CSF total homocysteine and CSF MMA could be considered as markers of early folate or B-12 functional deficiency at neuronal level.

Our aims in this study were to determine CSF levels of total homocysteine and MMA during aging and in Alzheimer's disease, and to determine whether an increase in total homocysteine and MMA concentration in the CSF could be implicated in the pathophysiology of Alzheimer's disease.

\section{METHODS}

\section{Patients}

Four groups of subjects were studied. In the first three, CSF samples were obtained by lumbar puncture done to rule out a neurological disease such as meningitis or subarachnoid haemorrhage or to carry out diagnostic myelography. In all CSF samples, protein levels were less than $0.60 \mathrm{~g} / \mathrm{l}$ and the cell count less than three cells/ml. CSF specimens were processed for their intended use and then frozen at $-60^{\circ}$.

The groups were as follows:

- group I, 33 subjects under 20 years of age (21 male, 12 female, mean age 8.9 years (range 1 to 20);

- group II, 28 subjects from 21 to 60 years of age ( 12 male, 16 female, mean age 40.5 years (range 23 to 58 ));

- group III, 22 subjects over 60 years of age (9 male, 13 female, mean age 75 years, range 60 to 89) with no evidence of cognitive impairment (mini-mental state examination (MMSE) score $>26$ );

- group IV, 38 patients with probable Alzheimer's disease over 60 years of age ( 15 male, 23 female, mean age 77.7 years (range 63 to 94)). The diagnosis of probable Alzheimer's disease was established according to the NINCDS-ADRDA criteria. In compliance with these criteria, all Alzheimer patients had normal CSF and normal serum vitamin B-12 levels; two patients had slightly reduced folate levels.

Abbreviations: MMA, methylmalonic acid; NINCDS-ADRDA, National Institute of Neurological and Communicative Disorders-Alzheimer's Disease and Related Disorders Association 

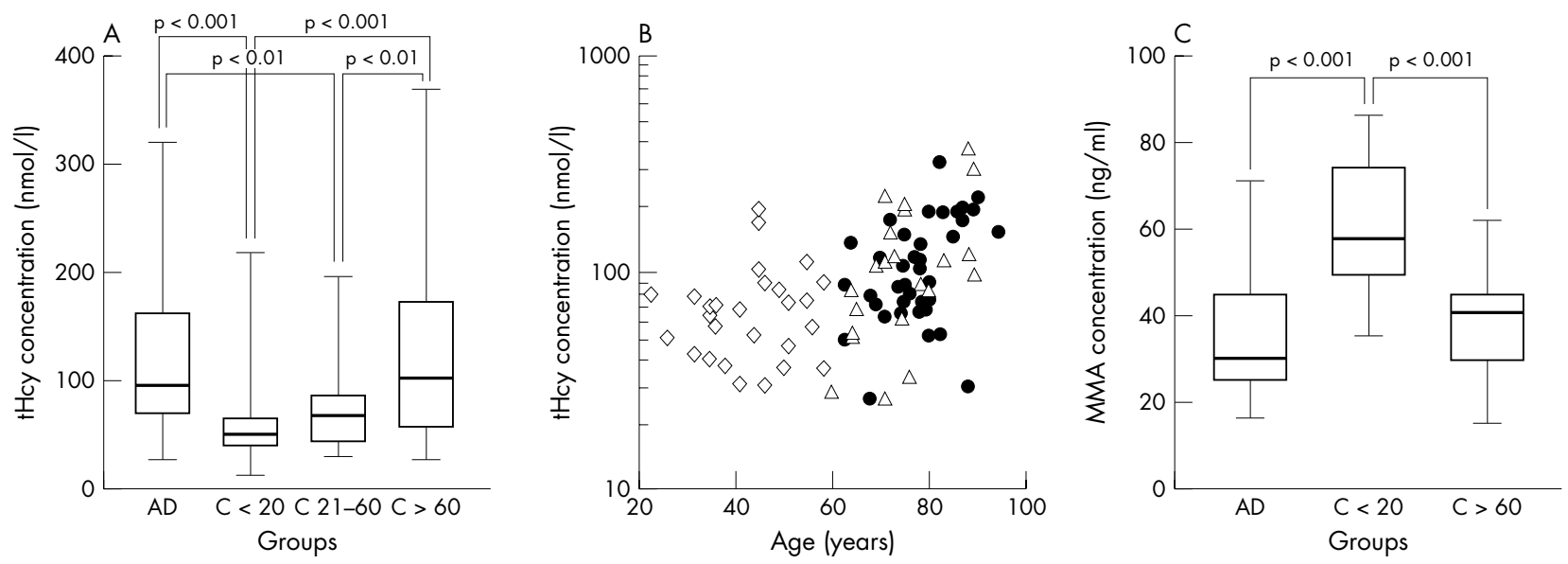

Figure 1 (A) Total homocysteine concentrations (min, max, median, 25th to 75th centile) in the patient groups: Alzheimer's disease patients over 60 years of age, controls (all mentally normal) under 20 years of age, from 21 to 60 years, and over 60 years of age. (B) Changes in total homocysteine concentration according to the age of subjects (filled circles, Alzheimer's disease patients over 60 years of age; empty diamonds, control mentally normal subjects from 21 to 60 years of age; empty triangles, elderly mentally normal subjects over 60 years of age). (C) MMA concentrations (min, max, median, 25th to 75th centile) in the patient groups, as for panel A. AD, Alzheimer's disease; C, control; tHcy, total homocysteine.

No patient had received drugs that could affect homocysteine, folate, or vitamin B-12 metabolism.

\section{Analyses \\ CSF homocysteine}

CSF total homocysteine was determined by high performance liquid chromatography (HPLC) coupled to fluorescence detection. ${ }^{10}$

\section{CSF MMA}

Cerebrospinal fluid MMA was measured by capillary gas chromatography-mass spectrometry. ${ }^{11}$

\section{Statistical analysis}

The computer program GraphPad Prism 3.00 (GraphPad Software, San Diego, California, USA) was used for all data analyses and statistics. The Gaussian distribution of the population was assessed by a Kolmogorov-Smimov test. Analysis of variance (ANOVA) with the Newman-Keuls post-test was undertaken for all group comparisons. The change in CSF homocysteine and MMA concentrations with age was quantified using Spearman correlation followed by linear regression.

\section{RESULTS}

In the youngest group $(<20$ years $)$, the mean (SD) concentration of total homocysteine in the CSF was 57 (35) $\mathrm{nmol} / \mathrm{l}$, significantly lower $(\mathrm{p}<0.001)$ than the mean concentration in the elderly control group (123 (89) nmol/l) or the Alzheimer group (115 (62) nmol/l) (fig lA). The mean concentration in the 21 to 60 year old group (72 (39) nmol/l) was lower $(p<0.01)$ than in the elderly group or the Alzheimer group (fig lA). The concentrations of total homocysteine in the elderly control group and in the Alzheimer group were not significantly different. The concentration of CSF homocysteine increased progressively with aging $(\mathrm{n}=87 ; r=0.4 ; \mathrm{p}<0.0001)$ (fig 1B).

The mean concentrations of CSF MMA were not significantly different in the elderly control group (38 (13) ng/ml) and the Alzheimer group (35 (14) ng/ml). In the youngest group, the concentration of CSF MMA was higher (at 60 (15) $\mathrm{ng} / \mathrm{ml} ; \mathrm{p}<0.001)$ than in the elderly control group or the Alzheimer group (fig 1C). Levels of CSF MMA fell significantly with age $(r=-0.5897, \mathrm{p}<0.0001)$.
In the Alzheimer group, no correlation was found between the serum folate and CSF homocysteine, or between serum vitamin B-12 and CSF MMA or CSF homocysteine.

\section{DISCUSSION}

Our results show a progressive increase in CSF total homocysteine and a decrease in CSF MMA in elderly subjects and patients with Alzheimer's disease. The decrease in CSF MMA was unexpected as serum levels have been found to increase with age. ${ }^{4}$

Homocysteine and MMA are neurotoxic and could be implicated in the pathogenesis of Alzheimer's disease. In vitro, neurones are sensitive to extracellular homocysteine. This amino acid promotes apoptosis in cultures of rat hippocampal neurones by induction of excitotoxicity, favours $\beta$ amyloid toxicity, induces oxidative stress, impairs endothelial function, and increases vascular medial thickness. ${ }^{12}$ MMA can induce neuronal damage in vitro, probably by non-specific stimulation of the NMDA receptor, allowing a rise in intracellular glutamate and thereby inducing apoptosis. ${ }^{12}$

Hyperhomocysteinaemia is a major vascular risk factor and is associated with an increased risk of silent brain infarction, stroke, and carotid atherosclerosis. ${ }^{13}$ The role of vascular factors seems important in the pathogenesis of Alzheimer's disease. ${ }^{14}$ Plasma homocysteine has been found to be increased in patients with vascular dementia and in histologically confirmed Alzheimer's disease..$^{15}$ In the present study, we found a similar increase in CSF total homocysteine levels in the elderly control group and in the Alzheimer's disease group; this suggests that the increase is not deleterious at a neuronal level in Alzheimer patients.

Impaired permeability of the blood-brain barrier could explain the raised CSF total homocysteine levels but not the decrease in CSF MMA that we found. In aging, folate and vitamin B-12 status is still a subject of discussion. ${ }^{471617}$ Several studies have shown that the serum and CSF levels of these vitamins are low in non-demented elderly people and in patients with Alzheimer's disease. However, vitamin and amino acid concentrations do not vary in parallel in the CSF and serum. The choroid plexuses are the blood brain-barrier site where active transport occurs for numerous molecules such as amino acids and vitamins $\mathrm{B}$ and $\mathrm{C}$, facilitated by specific carriers ${ }^{18}$ which are half saturated. In the case of serum deficiency, these carriers could be recruited to prevent variations in CSF levels. At this time, no homocysteine or 
MMA carrier have yet been described and no correlation between CSF and plasma total homocysteine, or between CSF and plasma MMA, has been found. ${ }^{3} 19$ The lack of carrier suggests that the brain is the chief origin of CSF MMA and CSF homocysteine. This hypothesis is supported by the fact that when methotrexate, an antifolate drug, is instilled into the ventricular cavity it induces profound changes in cerebrospinal fluid chemistry characterised by a rapid depletion of CSF folate followed by a marked increase in CSF total homocysteine. ${ }^{20}$

To interpret our results, it is also necessary to take into account the rate of secretion of CSF by the choroid plexuses. CSF flow is reduced in aging, and in Alzheimer's disease this decrease is greater. ${ }^{21}$ According to Rapoport et al, ${ }^{22}$ the combination of MMA (or homocysteine) levels and CSF flow may be more useful for studying the production of these brain metabolites than MMA (or homocysteine) levels alone. Our study suggests a decreased brain production and a lack of deficiency of folate or vitamin B-12 at neuronal level. This decline is in keeping with brain atrophy caused by aging or Alzheimer's disease.

\section{ACKNOWLEDGEMENTS}

This study was supported by a grant from the Fondation Jérôme Lejeune.

\author{
Authors' affiliations \\ J-M Serot, Department of Geriatric Medicine, Hôpital Sud, Amiens, \\ France \\ F Barbé, Laboratory of Biochemistry, Hôpital de Brabois, Vandoeuvre lès \\ Nancy, France \\ E Arning, T Bottiglieri, Baylor, University Medical Center, Institute of \\ Metabolic Disease, Dallas, Texas, USA \\ P Franck, Laboratory of Biochemistry, Hôpital Central, Nancy, France \\ P Montagne, Laboratory of Immunology, Faculty of Medicine, \\ Vandoeuvre lès Nancy, France \\ J-P Nicolas, Laboratory of Biochemistry, INSERM U724, Faculty of \\ Medicine, Vandoeuvre lès Nancy, France \\ Competing interests: none declared
}

Correspondence to: Dr J M Serot, Centre Saint Vincent, Hôpital Sud, Amiens 80054, France; serot.jean-marie@chu-amiens.fr

Received 12 October 2004

In revised form 2 March 2005

Accepted 2 March 2005

\section{REFERENCES}

1 Morris MS. Homocysteine and Alzheimer's disease. Lancet Neurol 2003;2:425-8.

2 Bolander-Gouaille C. Focus on homocysteine. The homocysteine metabolism. Springer-Verlag France, 2000:14-19.

3 Stabler SP, Allen RH, Barrett RE, et al. Cerebrospinal fluid methylmalonic acid levels in normal subjects and patients with cobalamin deficiency. Neurology 1991;41:1627-32.

4 Herrmann W, Schorr H, Bodis M, et al. Role of homocysteine, cystathionine and methylmalonic acid measurement for diagnosis of vitamin deficiency in high-aged subjects. Eur J Clin Invest 2000;30:1083-9.

5 Joosten E, Lesaffre E, Riezler R, et al. Is metabolic evidence for vitamin B-12 and folate deficiency more frequent in elderly patients with Alzheimer's disease? J Gerontol A Biol Sci Med Sci 1997:52:M76-9.

6 Blom HJ, Wevers RA, Verrips A, et al. Cerebrospinal fluid homocysteine and the cobalamin status of the brain. J Inherit Metab Dis 1993;16:517-19.

7 Ikeda T, Furukawa Y, Mashimoto S, et al. Vitamin B12 levels in serum and cerebrospinal fluid of people with Alzheimer's disease. Acta Psychiatr Scand 1990;82:327-9.

8 Bottiglieri T, Reynolds EH, Laundy M. Folate in CSF and age. J Neurol Neurosurg Psychiatry 2000;69:562.

9 Serot JM, Christmann D, Dubost T, et al. CSF-folate levels are decreased in late-onset AD patients. J Neural Transm 2001;108:93-9.

10 Ubbink JB, Hayward Vermaak WJ, et al. Rapid high-performance liquid chromatographic assay for total homocysteine levels in human serum. J Chromatogr 1991;565:441-6.

11 Straczek J, Felden F, Dousset B, et al. Quantification of methylmalonic acid in serum measured by capillary gas chromatography-mass spectrometry as tert.butyldimethylsilyl derivatives. J Chromatogr 1993;620:1-7.

12 Kolker S, Ahlemeyer B, Krieglstein J, et al. Methylmalonic acid induces excitotoxic neuronal damage in vitro. J Inherit Metab Dis 2000;23:355-8.

13 Matsui T, Arai H, Yuzuriha T, et al. Elevated plasma homocysteine levels and risk of silent brain infarction in elderly people. Stroke 2001;32:1116-19.

14 de la Torre JC. Vascular basis of Alzheimer's pathogenesis. Ann NY Acad Sci 2002;977:196-215.

15 McCaddon A, Regland B, Hudson P, et al. Functional vitamin B(12) deficiency and Alzheimer disease. Neurology 2002;58:1395-9.

16 Clarke $R$, Smith $A D$, Jobst $K A$, et al. Folate, vitamin $B 12$, and serum total homocysteine levels in confirmed Alzheimer disease. Arch Neurol 1998;55: 1449-55.

17 Nijst TQ, Wevers RA, Schoonderwaldt $\mathrm{HC}$, et al. Vitamin $\mathrm{B} 12$ and folate concentrations in serum and cerebrospinal fluid of neurological patients with special reference to multiple sclerosis and dementia. J Neurol Neurosurg special reference to multiple
Psychiatry 1990;53:951-4

18 Serot JM, Bene MC, Faure GC. Choroid plexus, ageing of the brain, and Alzheimer's disease. Front Biosci 2003;8:S515-21.

19 Vrethem M, Mattson E, Hebelka H, et al. Increased plasma homocysteine levels without signs of vitamin B12 deficiency in patients with multiple sclerosis assessed by blood and cerebrospinal fluid homocysteine and methylmalonic acid. Multiple Sclerosis 2003;9:239-45

20 Quinn CT, Griener JC, Bottiglieri T, et al. Effects of intraventricular methotrexate on folate, adenosine, and homocysteine metabolism in cerebrospinal fluid. J Pediatr Hematol Oncol 2004;26:386-8.

21 Silverberg GD, Heit G, Huhn S, et al. The cerebrospinal fluid production rate is reduced in dementia of the Alzheimer's type. Neurology 2001;57:1763-6.

22 Rapoport SI, Schapiro MB, May C. Reduced brain delivery of homovanillic acid to cerebrospinal fluid during human aging. Arch Neurol 2004;61:1721-4 